reply) and percentage discrimination (i.e. difference in facility between top and fourth quartile of students).

Each of the eight Sections is subdivided so as to offer a choice of MCQs of the following types: A, True or false questions (if true, are the clauses related?); B, The most appropriate statement (to be selected from four choices); C, Comparative questions; $\mathrm{D}$, All appropriate statements from 0 to 4 may be correct and E, Matching questions. The directions to students are of assistance but the paragraph on comparative statements, even when taken in conjunction with the comparative MCQs and the answers, could lead to residual uncertainty.

As there are various types of MCQs, the author has given groups of each type within each Section. The type of MCQ used by Teachers and Examiners tends to crystallize into one or two types so that the other three types might be of little relevance to many students. The shortcoming of the laudable attempt to be all-embracing is that the numbers of examples of MCQs of each type are limited to about 150 MCQs. Most candidates can answer up to $60 \mathrm{MCQs}$ comfortably in one hour. If examples of MCQ are required in one or more types, then the book can provide them, but it is tempting to enquire whether the number offered in each type is adequate.

It is very easy to criticize MCQs, it is very much harder to compose MCQs and to assess critically the products of one's composition. Therefore we can be grateful to Professor Lewis for tackling the task. This book should stimulate student understanding of and interest in MCQ techniques. As MCQs seem to have become an established feature of the examination scene, no student sitting examinations with MCQs can afford to be less than well prepared and this book offers help in this direction.

\section{Multiple Sclerosis: British Medical Bulletin, Vol. 33, No. 1}

Pp. $85+$ xiv, soft cover, illustrated. London: The British Council, Medical Department, 65 Davies Street, 1977. $£ 4.50$ UK. $£ 5.00$ Other countries.

This up-to-date review of current knowledge on multiple sclerosis deals with its pathology, epidemiology, clinical features, laboratory diagnosis and management. The relevance of viral antibody studies, histocompatibility antigens, fatty acids, cellular immunity and humoral factors are discussed by experts in the respective fields. None of the fourteen contributions is more than seven pages long and the whole, edited by J. S. Porterfield, will be invaluable as well as of considerable interest both to clinicians and research workers in this field.

\section{Multiple sclerosis. A critical conspectus}

Edited by E. J. Field. Pp. 265, hardback, illustrated. Lancaster: MTP Press, 1977. £9.95.

Professor Field has brought together a number of scientists concerned with research into multiple sclerosis and has succeeded in editing an excellent book on the subject. Although almost half of it is composed of two chapters on epidemiology, the authors Alter and Kurtzke have succeede in avoiding significant overlap. Kurtzke's chapter is of con@ siderable interest in its review of the pitfalls of data collectione. In the remaining half of the book Fog deals with the clinicat aspects of the disease, Carp and his associates with the sige? nificance of the recent viral studies, Smith and Thompsops with those on lipids and Carnegie and Sims with proteins and enzymes. Changes in the CSF are discussed by Lowenthal Field himself contributes the introductory chapter and in the final one discusses the significance of the tests for the diseas which he has been instrumental in devising and which have given rise to a certain amount of controversy.

\section{Operative Obstetrics (9th Edn)}

By P. R. Myerscough. Pp. $882+$ ix, hard cover, illus $\vec{\omega}$ trated. London: Baillière Tindall, 1977. $£ 15.00$ cased.

This is the ninth edition of a great book, going back ovee seventy years. Munro Kerr started it, Chassar Moir con? tinued it and Philip Myerscough has been an editor sincer 1971. It is a very personal volume, depending heavily on the practice of these three men and so must be a very reassuring book to have beside one when in trouble. It presents a wide survey of the operative management of paranormal obstet $\vec{\omega}$ rics. Parts of it seem like another world to the obstetrician of the late 1970s working in the United Kingdom, but there arè still large areas of the world where a volume like this would be invaluable to the single-handed obstetrician meeting a condition for the first time and having limited facilities with which to deal with it. Dr Myerscough has retained theD personal style of his previous senior authors including many anecdotal accounts of cases dealt with in the past.

Accepting this, the book does tend to hark back and the references quoted have the look of a vintage port list ratger than one of usual everyday wines; there is a distinct skewt the left. For instance, in the references associated with diagnosis of pelvic deformity, all but two are pre-1970 apd many of the references to the management of breech deliveryo are from World War II or before; there have been many more modern references which deserve recommendation. This is matter of judgment for it is very difficult to follow great mem of the past and not acknowledge what they have done. If however, in the next edition, this could be edited to a slightly wider acceptance of obstetrics in the last twenty years, is would make the book more in line with what happens in the labour wards of the United Kingdom in the 1970s and we hope the 1980s.

The book is thoroughly recommended to all who practise. obstetrics. It will teach us all something, for the authors draw on a wealth of practice which between the three of them spans a century of work. Operative Obstetrics is an essential for alt. medical libraries and should certainly be on the shelf of any 3 obstetrician who may find himself not working in the safer surroundings of the home labour wards and who may haveo to resort to a variety of vaginal procedures for which his Western training had not fitted him. 